\title{
Efectos clínicos a largo plazo en niños intoxicados con plomo en una región del sur de Chile
}

\author{
Carolina Coria ${ }^{1,5}$, Aurora Cabello 2,5 , Enzo Tassara ${ }^{2,5}$, \\ Eduardo López ${ }^{1,5}$, H ugo Rosales ${ }^{1}$, Marcelo Pérez ${ }^{1}$, \\ Claudia Zavala 3,5 , Patricio Muñoz ${ }^{1}$, Gabriel 0 rellana ${ }^{4 a}$, \\ María Isabel Inostroza ${ }^{4 a}$, Lorena Contreras ${ }^{4 a}$, Lilian Kirsten ${ }^{5 b}$. \\ Long term consequences among \\ children exposed to lead poisoning
}

Background: In 1996 there was a massive lead poisoning in a southern rural community in Chile. The contamination source was a mill whose grinding stone was repaired with lead and contaminated the flour. Aim: To assess the presence of sequelae ten years later, among subjects that were exposed to lead on that occasion. Material and methods: Cross sectional study of 77 individuals (47 males), aged 10 to 25 years, that were exposed to lead in 1996 and were treated with EDTA. Results: Twenty one percent of subjects had a subnormal intellingence quotient (IQ). The risk of having a low IQ was significantly higher among those exposed before the age of six years. IQ was significantly lower among subjects that, immediately after the exposure, had a lead level over $48 \mu \mathrm{g} / \mathrm{dl}$, compared with those that had a lead level below $43 \mu \mathrm{g} / \mathrm{dl}(86.7 \pm 7.3$ and $93 \pm 11.6$ respectively). No subjects with high blood pressure or evidences of nephrotoxicity were detected. Conclusions: Subjects aged less than six years at the moment of lead exposure had a lower IQ when assessed ten years later (Rev Méd Chile 2009; 137: 1037-44).

(Key w ords: Intelligence; Lead poisoning, nervous system; Neurotoxicity syndromes)

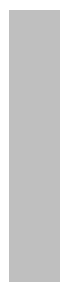

Recibido el 18 de junio, 2008. Aceptado el 15 de julio, 2009.

Trabajo financiado por Proyecto de Investigación DIN - Dirección de Investigación y Postgrado de la Universidad Católica de la Santísima Concepción.

${ }^{1}$ Servicios de Neurología, ${ }^{2}$ Pediatría, ${ }^{3}$ Medicina Interna y ${ }^{4}$ Consultorio Adosado de Especialidades del Hospital Clínico Herminda Martín, Chillán. ${ }^{5}$ Facultad de Medicina, Universidad Católica de la Santísima Concepción (UCSC), Concepción.

apsicólogo Clínico

bMaster en Bioestadística

$\mathrm{E}$ 1 plomo es un metal pesado que se encuentra ampliamente distribuido en la corteza terrestre en forma natural o como consecuencia de su

Correspondencia a: Dra. Carolina Coria De la Hoz. Constitución 381, Chillán. Fax: 42-222367. E mail: ccoria@ucsc.cl empleo industrial. Fue reconocido como agente tóxico hace tres milenios y su efecto en la población infantil se conoce hace aproximadamente cien años, cuando Gibson describió la intoxicación plúmbica en niños por ingestión de pinturas en base a plomo ${ }^{1}$. El plomo es un neurotóxico muy potente, que puede generar efectos adversos en varias vías neuroquímicas, 
alterando la integridad de la barrera hematoencefálica, sinaptogénesis, mielinización y metabolismo de catecolaminas en el sistema nervioso central (SNC) ${ }^{2}$. La intoxicación grave se caracteriza por dolor abdominal cólico, anemia y encefalopatía, y ocurre con niveles plasmáticos de plomo sobre $40 \mu \mathrm{g} / \mathrm{dl}$, generando mortalidad sobre $70 \mu \mathrm{g} / \mathrm{dl}$. Los niños que sobreviven quedan con importantes secuelas cognitivas ${ }^{3}$. Niveles menores de plumbemia también se asocian a retraso mental (RM) o problemas conductuales ${ }^{4}$.

La exposición al plomo es un problema de salud pública tanto en centros urbanos de países desarrollados como aquellos en vías de desarrollo. En Chile se han detectado poblaciones afectadas en ciudades como Arica y Antofagasta, debido a focos de contaminación ambiental procedentes de acopios de desechos tóxicos ${ }^{5}$.

En relación a los efectos del plomo en población infantil se ha demostrado la vulnerabilidad del cerebro en desarrollo, el cual puede sufrir daños significativos y a menudo irreversibles, evidenciándose alteraciones neuropsiquiátricas con niveles incluso menores de $10 \mu \mathrm{g} / \mathrm{dl} l^{6,7}$.

En 1996 en una comuna rural de Ñuble ocurrió una intoxicación masiva por plomo. La fuente contaminante fue un molino de trigo cuya piedra de molienda había sido reparada con plomo, el cual al desprenderse contaminaba las harinas que abastecían a la población de la comuna. El primer caso diagnosticado fue una niña de 6 años que se hospitalizó por desnutrición, dolor abdominal, anemia y encefalopatía progresiva, falleciendo a los 4 días del ingreso. La presencia de anemia grave y un punteado basófilo en los glóbulos rojos hizo sospechar una intoxicación plúmbica, confirmándose una plumbemia de $104 \mu \mathrm{g} / \mathrm{dl}$. De 2.056 personas expuestas, $40 \%$ eran menores de 15 años 8 . Ciento cuarenta niños tenían plumbemia muy alta (37 a $114 \mu \mathrm{g} / \mathrm{dl}$ ), por lo que recibieron tratamiento quelante con EDTA cálcico.

El objetivo de esta investigación fue evaluar, diez años después de la intoxicación, el impacto que ésta tuvo en los aspectos biomédicos, neurológicos y cognitivos en el grupo de niños que recibieron tratamiento quelante.

\section{PACIENTES Y MÉTODO}

Estudio transversal descriptivo de una cohorte histórica de una población pediátrica ( $\leq 15$ años) de la comuna de San Ignacio (Ñuble, Octava Región) expuesta a una intoxicación plúmbica masiva en 1996 y tratada con EDTA cálcico.

La evaluación para este estudio se realizó en el Hospital de Chillán, entre agosto y octubre de 2006, previa información de los objetivos a cada paciente o a su tutor en menores de edad, y la firma del consentimiento informado.

Población estudiada. De los 140 pacientes que se habían tratado por la intoxicación, se logró ubicar a 78. Se excluyó un paciente de 18 años, que rechazó participar en el estudio, quien en 1996 presentó una polineuropatía sensitivo-motora axonal secundaria a la intoxicación (plumbemia de 88 $\mu \mathrm{g} / \mathrm{dl}$ ). Se estudiaron 77 pacientes ( 47 hombres y 30 mujeres), con una edad promedio de 18,3 años (entre 10-25 años).

Método de evaluación. Se consignaron los datos sociodemográficos: edad, sexo, escolaridad, inserción laboral, ingresos económicos y antecedentes mórbidos. Se realizó examen físico por pediatra y neuropediatra en menores de 18 años, y por médico internista y neurólogo en los de 18 o más. Se midió la velocidad de conducción nerviosa (VCN) (equipo Nicolet VikingQuest), y se determinó el coeficiente intelectual (CI) mediante una psicometría. Los exámenes de laboratorio realizados fueron: hemograma, triglicéridos, colesterol total, uricemia, bilirrubinemia, calcemia, uremia, creatininemia, glicemia, fosfatasas alcalinas, transaminasas (GPT, GOT) y gammaglutamiltranspeptidasa (GGT). Además se midió plomo en sangre por el método de absorción atómica en horno de grafito?

El estudio de psicometría se efectuó mediante dos tests: WISC-R $(<16 \text { años })^{10}$ y WAIS $(\geq 16$ años $)^{11}$, y se clasificaron los pacientes de acuerdo al puntaje obtenido en: CI normal superior ( $>110)$, CI normal promedio (90-109), CI normal lento (80-89), CI limítrofe (70-79), RM leve (50-69), RM moderado (40-49), RM severo (20-39). Se relacionó el CI con el nivel de plomo histórico y con la edad de exposición. Para este análisis se excluyeron 3 pacientes: dos con RM familiar y uno con 
deterioro neurológico global, secuela de un traumatismo encéfalo-craneano (TEC) grave.

Estadística. Los datos obtenidos se analizaron con el programa STATISTICA 6.0. Se utilizó chi-cuadrado para estudiar asociaciones entre variables, $t$ de Student para la comparación de medias de muestras no pareadas, comparación de proporciones en muestras independientes y para el análisis univariado se calculó el coeficiente de correlación de Pearson. Se estableció un valor alfa de 0,05 como punto de corte para la significación estadística.

\section{Resultados}

El nivel educacional y socioeconómico de los grupos familiares de los pacientes fue homogéneo, con $14 \%$ de analfabetismo y promedio de escolaridad de 6,5 años. El nivel socioeconómico fue bajo en $76,6 \%$, medio bajo en $20,8 \%$ y medio alto en $2,6 \%$.

Para el análisis de escolaridad e inserción laboral se estratificó la muestra en dos grupos de edad (Tabla 1). Un grupo estuvo compuesto por los menores de 18 años $(n=38)$, que les correspondía escolaridad básica (EB) o media (EM) y un segundo grupo de 18 años o más $(\mathrm{n}=39)$. Del grupo $<18$ años, sólo 19 (50\%) estaban en un curso acorde a la edad cronológica. Del grupo $\geq 18$ años, 8 (20\%) aún estaban en etapa escolar. De los 31 restantes, 5 desertaron ( 3 en EB y 2 en EM) y de los 26 que continuaron estudios, 3 completaron EB y 23 finalizaron EM. Nueve de estos últimos continuaron estudios superiores (6 técni- cos y 3 universitarios). Trece (33\%) tienen trabajo remunerado, cuatro $(10,2 \%)$ estudian y trabajan, y nueve $(23 \%)$ no estudian ni trabajan.

De los 77 pacientes, 30 (39\%) ha presentado al menos una repitencia de curso posterior a 1996, y 18 (23\%) dos o más repitencias, lo cual es mayor a lo esperado de acuerdo a datos disponibles a nivel nacional ${ }^{12}$.

Examen físico. El 33,7\% de la población estudiada presentaba algún tipo de anormalidad, siendo las más frecuentes, en orden decreciente: lesiones dermatológicas (acné, dermatitis, secuela de quemadura, rosácea) en $14,2 \%$, sobrepeso $(10,3 \%)$, obesidad $(6,4 \%)$, acantosis nigricans $(5,1 \%)$, hipertensión arterial sistólica (3,8\%), pie cavo $(2,5 \%)$, soplo cardíaco $(2,5 \%)$, onicomicosis $(2,5 \%)$, escoliosis $(1,2 \%)$ y nódulos mamarios $(1,2 \%)$.

Examen neurológico. El 54,5\%, presentó alguna alteración en el examen neurológico, apareciendo entre las principales: el examen mental en 30\% (memoria, abstracción), el examen motor en 10\% (hipo o arreflexia, hipotrofia distal), el examen sensitivo superficial en $10 \%$, sensibilidad profunda en $6,4 \%$, y el examen cerebeloso (temblor de intención) en $6,4 \%$. Un paciente evidenciaba hemiparesia y deterioro cognitivo como secuela de TEC grave.

Estudio de VCN. Se realizó en 71 pacientes (92\%), encontrándose alteraciones en 5 de ellos (6,5\%): mononeuropatía axonal del nervio tibial (2), túnel del carpo sensitivo derecho (1), atrapamiento cubital: derecho (1), bilateral (1).

Tabla 1. D atos de escolaridad

\begin{tabular}{|lccc|}
\hline & $\begin{array}{c}\text { Menores de 18 años } \\
(\mathbf{n}=\mathbf{3 8})\end{array}$ & $\begin{array}{c}\text { Mayores de 18 años } \\
\text { (n =39) }\end{array}$ & Total \\
\hline En enseñanza básica (EB) & 10 & 1 & 11 \\
En enseñanza media (EM) & 23 & 7 & 30 \\
Deserción escolar en EB & 0 & 3 & 3 \\
Deserción escolar en EM & 5 & 2 & 7 \\
Completaron EB & 4 & 3 & 7 \\
Completaron EM & 0 & 23 & 23 \\
\hline
\end{tabular}


Análisis de sangre. No se evidenció anemia en ningún paciente. Siete presentaron hiperlipidemia (9\%), cuatro aumento de la GPT $(5,2 \%)$, tres de la GGT (3,9\%), uno aumento de la GOT y uno hiperbilirrubinemia indirecta. La función renal fue normal en todos.

Los resultados de plumbemia descartaron una intoxicación actual (niveles entre 0-13 $\mu / \mathrm{dl}$ ).

Psicometría. Se realizó en 76 pacientes. La Tabla 2 muestra la distribución de la muestra en relación al rango de CI, observándose que 1,3\% era normal superior, 39,4\% normal promedio y $36,8 \%$ normal lento. El 22,49\% restante mostró alguna alteración en el CI: $11,8 \%$ CI limítrofe, 9,2\% RM leve y 1,3\% RM moderado.

No se observaron diferencias significativas al comparar el diagnóstico de CI entre los diferentes grupos socioeconómicos ( $\mathrm{p}=0,111$ ).

CI y edad de exposición. La edad de exposición al plomo y el nivel intelectual se asocian en forma significativa ( $p=0,017$ ) (Tabla 3). La posibilidad de tener un CI subnormal en aquellos niños intoxicados con plomo antes de los 6 años resultó significativamente mayor en comparación con los intoxicados después de los 6 años (OR =6,53 (IC: 1,9-22,9) p <0,003).

Uno de los pacientes con RM tenía 3 meses de vida en el período de la intoxicación y se alimentaba con lactancia materna exclusiva (nivel de plomo histórico de $44 \mu \mathrm{g} / \mathrm{dl}$ ).

CI y nivel de plomo histórico (1996). No hubo correlación entre el nivel de plomo histórico y el puntaje de CI actual. Sin embargo al estratifi-
Tabla 2. D istribución de frecuencia de nivel de CI

\begin{tabular}{|lrr|}
\hline N ivel intelectual & $\mathbf{n}$ & $\%$ \\
\hline CI normal superior & 1 & 1,31 \\
CI normal promedio & 30 & 39,4 \\
CI normal lento & 28 & 36,8 \\
Limítrofe & 9 & 11,8 \\
RM leve & 7 & 9,2 \\
RM moderado & 1 & 1,31 \\
Total & 76 & 100 \\
\hline
\end{tabular}

car el nivel de plomo histórico en cuartiles y comparar entre ellos los puntajes promedio de CI, se encontró diferencias significativas al comparar el cuartil con niveles más bajos de plomo $(37-42 \mu \mathrm{g} / \mathrm{dl})$ con los dos más altos (48-56 y 57$87 \mu \mathrm{g} / \mathrm{dl}, \mathrm{p}=0,0039$ y $\mathrm{p}=0,0482$, respectivamente) (Tabla 4).

Subpruebas WISC-R y WAIS. Se analizó en la muestra si existía alguna subprueba de los tests psicométricos cuyo puntaje estuviera significativamente por debajo de las otras, independiente del puntaje de CI total. En cada subprueba se determinó el porcentaje de pacientes con un puntaje significativamente inferior. Al comparar ambos grupos (WISC-R y WAIS), se vio una mayor proporción de pacientes con resultados disarmónicos en el grupo de mayor edad (WAIS), lo que resultó estadísticamente significativo en las subpruebas de aritmética y dígitos (Tabla 5).

Tabla 3. Edad de exposición (menor y mayor de 6 años) versus nivel intelectual

\begin{tabular}{|c|c|c|c|c|c|}
\hline \multirow[t]{2}{*}{ Edad de exposición } & \multicolumn{2}{|c|}{$\begin{array}{c}n \text { voluntarios } \\
\mathrm{CI} \text { normal y normal Iento }\end{array}$} & \multicolumn{2}{|c|}{$\begin{array}{l}\text { n voluntarios con RM } \\
\text { y CI limítrofe }\end{array}$} & \multirow[t]{2}{*}{ Total } \\
\hline & $n$ & $\%$ & n & $\%$ & \\
\hline $0-5$ años $11 \mathrm{~m}$ & 10 & 55,5 & 8 & 44,5 & 18 \\
\hline 6 a 15 años & 49 & 89 & 6 & 11 & 55 \\
\hline Total & 59 & & 14 & & 73 \\
\hline
\end{tabular}

Prueba de Chi cuadrado $\mathrm{p}=0,017$. 
Tabla 4. Nivel de plomo (1996) y coeficiente intelectual

\begin{tabular}{|c|c|c|c|c|}
\hline \multirow[b]{2}{*}{ Cuartil } & \multicolumn{2}{|c|}{ Nivel plomo 1996 ( $\mu \mathrm{g} / \mathrm{dl})$} & \multicolumn{2}{|c|}{ C oeficiente intelectual (puntos) } \\
\hline & rango & $\overline{\mathbf{x}} \pm \mathrm{DS}$ & rango & $\overline{\mathrm{x}} \pm \mathrm{DS}$ \\
\hline$(\mathrm{n}=19)$ & $37-42$ & $40 \pm 1,34$ & $64-112$ & $93 \pm 11,58$ \\
\hline$(n=16)$ & $43-47$ & $45 \pm 1,61$ & $60-105$ & $84,8 \pm 13,6$ \\
\hline III $\quad(n=17)$ & $48-56$ & $50,9 \pm 2,68$ & $69-103$ & $85,41 \pm 9,4$ \\
\hline IV $\quad(n=20)$ & $57-87$ & $66,4 \pm 9,09$ & $68-99$ & $86,7 \pm 7,30$ \\
\hline
\end{tabular}

Tabla 5. Porcentaje de pacientes con puntaje significativamente inferior en una subprueba del test psicométrico

\begin{tabular}{|lcccc|}
\hline Subpruebas & $\begin{array}{c}\text { WISC-R } \\
(\mathbf{n}=\mathbf{2 0})\end{array}$ & \% & $\begin{array}{c}\text { WAIS } \\
\text { (n =55) }\end{array}$ & $\%$ \\
\hline Comprensión & 4 & 20 & 6 & 10,9 \\
Aritmética & 2 & $10^{*}$ & 28 & $50,9^{*}$ \\
Semejanzas & 0 & 0 & 1 & 1,81 \\
Dígitos & 0 & $0^{* *}$ & 13 & $23,63^{* *}$ \\
Claves & 2 & 10 & 6 & 10,9 \\
Completación & 0 & 0 & 2 & 3,63 \\
Cubos & 0 & 0 & 2 & 3,63 \\
Ordenamiento historietas & 1 & 5 & 4 & 7,27 \\
\hline
\end{tabular}

*p $=0,031 . \quad * * \mathrm{p}=0,034$

\section{DISCUSIÓN}

A pesar de las medidas que a nivel mundial se han implementado para la prevención de la intoxicación plúmbica, la exposición a este tóxico continúa siendo un problema de salud pública ${ }^{1}$. Existen diferencias en la contaminación con plomo entre niños y adultos, tales como: el modo de exposición, vías de metabolismo y toxicidad ${ }^{13}$. Los niños pequeños exploran el ambiente vía mano-boca, por lo que son más susceptibles a la contaminación ambiental (pinturas con plomo, polvo, baterías), y la fracción de plomo que absorben a nivel gastrointestinal es mucho mayor que en los adultos, incrementándose en presencia de deficiencias nutricionales que también son más frecuentes en la infancia ${ }^{14}$. Otra forma de intoxicación es el traspaso de plomo desde la madre por vía transplacentaria o a través de la lactancia, como se observó en uno de los casos del estudio. Este mecanismo está documentado en la literatura donde se enfatiza el riesgo para el recién nacido ${ }^{15}$.

Tanto en niños como en adultos, la intoxicación por plomo afecta a múltiples órganos. Si bien en el adulto genera anormalidades en el SNC, tiende a predominar la neuropatía periférica ${ }^{16-18}$, que puede ser reversible. En el niño, en cambio, los efectos centrales son más significativos que los periféricos y permanecen en el tiempo, con importantes secuelas en la esfera neuropsiquiátri$\mathrm{ca}^{16,19}$, aun después de haber recibido terapia quelante ${ }^{20,21}$. En nuestro estudio no se demostró compromiso neuropático como el descrito en la intoxicación plúmbica, ya que los resultados del estudio de conducción nerviosa son posibles de 
encontrar en la población general. Sólo en el paciente que rechazó la evaluación, se confirmó una polineuropatía como efecto inmediato a la intoxicación.

La alta asociación observada entre la menor edad de exposición al tóxico y el menor rendimiento cognitivo, junto a la significativa mayor posibilidad de CI subnormal en los niños intoxicados antes de los 6 años, confirma el efecto más deletéreo del plomo en el cerebro en desarrollo. Se ha postulado que este efecto tóxico es multifactorial debido a un incremento de la apoptosis neuronal y excitotoxicidad, anormalidades en la función mitocondrial, disrupción del sistema endotelial cerebrovascular, daño oligodendroglial y de conexiones sinápticas con alteración en la neurotransmisión glutamaérgica y dopaminérgica afectando el aprendizaje, memoria, control motor, atención y función ejecutiva ${ }^{22-24}$.

La relación del nivel de plomo y el CI ha mostrado resultados contradictorios en la literatura, pero en general se acepta que existe una relación inversa entre ambas variables ${ }^{22}$, tomando en cuenta que existen factores confundentes en esta relación, como el factor socioeconómico. En nuestro estudio los grupos de niños con niveles más altos de plumbemia histórica tuvieron puntajes significativamente menores de CI. La condición socioeconómica no se asoció significativamente con el CI, lo que confirma el nivel de intoxicación como factor determinante en los bajos puntajes psicométricos obtenidos.

En el estudio psicométrico destaca el menor rendimiento observado en pruebas de aritmética y dígitos en los pacientes evaluados con WAIS, a diferencia del grupo WISC-R, quienes presentan un rendimiento más armónico entre las subpruebas. Las subpruebas de aritmética y dígitos se relacionan con la atención, concentración y memoria de trabajo, funciones que residen principalmente en el lóbulo frontal, donde la corteza prefrontal tiene un rol preponderante. Ésta se desarrolla tardíamente en la ontogenia del sistema nervioso, alcanzando su madurez completa en la adolescencia $^{25}$. Esto podría explicar el compromiso selectivo de estas funciones en los niños mayores del estudio, apoyando el concepto de período crítico o ventanas de oportunidad en el neurodesarrollo. Se ha visto en estudios de exposición al plomo, mayor alteración en la sustancia gris frontal mediante espectroscopia de resonancia magnética ${ }^{26}$, como también el compromiso selectivo del razonamiento aritmético ${ }^{27}$.

Se han descrito otros efectos deletéreos sistémicos asociados a la intoxicación plúmbica, como la enfermedad cardiovascular, pérdida dentaria, retraso puberal, menor fertilidad, aborto espontáneo, enfermedad renal y cataratas ${ }^{28-36}$. Ninguno de ellos se observaron en la evaluación actual, pero deberá mantenerse un seguimiento estrecho de esta cohorte, ya que algunos efectos pudieran observarse a largo plazo, especialmente la hipertensión arterial y el daño renal, lo cual aumenta el riesgo cardiovascular si se agrega a otros de prevalencia creciente observados en la población chilena como diabetes, tabaquismo, vida sedentaria y obesidad ${ }^{37}$.

Dentro de las limitaciones de este estudio destaca el desconocimiento de la evolución clínica y neurológica del resto de los niños que recibieron tratamiento quelante y que no se lograron ubicar, cuya información enriquecería el análisis y conclusiones de esta investigación. Por otro lado, en estudios posteriores sería de utilidad comparar los resultados de los CI obtenidos con la distribución normal de CI en poblaciones rurales similares.

En conclusión, los hallazgos observados en relación al menor rendimiento cognitivo y la menor edad de exposición al plomo, refuerzan la necesidad de establecer políticas de salud pública de tamizaje de agentes neurotóxicos (plomo, mercurio o arsénico) especialmente en la población infantil donde el efecto deletéreo es mayor e irreversible. Asimismo se requiere desarrollar acciones de prevención en salud ambiental, y mantener un alto grado de sospecha diagnóstica tanto de la intoxicación plúmbica aguda como en casos crónicos que pueden cursar con retraso del desarrollo psicomotor, déficit atencional o problemas de aprendizaje.

\section{Agradecimientos}

- Laboratorio de Higiene Industrial de la Asociación Chilena de Seguridad (medición de plomo en sangre).

- Sra. Viviana Bustamante, directora del Consultorio de la comuna de San Ignacio y a todo el personal del consultorio que colaboró en la citación de los pacientes.

- Dra. Pilar Alonso por el aporte de material y datos epidemiológicos.

- Dr. Carlos Zúñiga S.M. y Dra. Paulina Coria por su aporte en la revisión del manuscrito. 


\section{Referencias}

1. LANPhEar B. Childhood lead poisoning prevention. JAMA 2005; 293: 2274-6.

2. Deitrich K. Enviromental neurotoxicants and psychological development. En: K.O Yeats, M.D. Ris and H.G. Taylor, Editors. Pediatric neuropsychology: research, theory and practice. Guilford Press, London (2000), 206-34.

3. Cory-Schlecta DA, Schaumburg HH. Lead, inorganic. En: Spencer PS, Schaumburg HH, Ludolph AC, editors. Experimental and clinical neurotoxicology. $2^{\text {nd }}$ ed. New York: Oxford University Press, 2000; 708-20.

4. Chen A, Cai B, Dietrich K, Radcliffe J, Rogan W. Lead exposure, IQ, and behaviour in urban 5-7 year olds: Does lead affect behaviour only by lowering IQ? Pediatrics 2007; 119: e650-e658.

5. Tchernitchin A, Lapin N, Molina L, Molina G, Tchernitchin N, Acevedo C, Alonso P. Human Exposure to lead in Chile. Rev Environ Contam Toxicol 2006; 185: 93-139.

6. Canfield R, Henderson CR, Cory-Slechta D, Cox C, Jusko TA, LANPhear B. Intellectual impairment in children with blood lead concentrations below 10 micrograms per deciliter. N Engl J Med 2003; 348 : 1517-26.

7. Rogan W, Ware J. Exposure to lead in children: How low is low enough? N Engl J Med 2003; 348: 1515-6.

8. Alonso P, Castro H, Davis me, Goza J, Hargous J, Rivera L, Tassara E. Intoxicación por plomo: estudio epidemiológico Ñuble, Chile. EPI Visión, Ministerio de Salud, Chile, 1997; 10-5.

9. Fernández FJ. Micromethod for lead determination in whole blood by atomic absorption with use of the graphite furnace. Clin Chem 1975; 21: 558-61.

10. WeChSLER D. Test de inteligencia para niños WISC III. Manual. Psicometría y diagnóstico. Ed. Paidos, abril 1999.

11. Wechsler D. Test de inteligencia para adultos WAIS. Manual. Psicometría y pasicodiagnóstico. Ed. Paidos, febrero 1992

12. MINEDUC. Compendio de información estadística educacional 1999-2000. www.cep.cl/Cenda/Proyectos/Colegio_Profesores/Referencias/UNICEF/ Cobertura.pdf fecha de consulta 10 noviembre 2008.

13. Bellinger D. Lead. Pediatrics 2004; 113: 1016-22.

14. Bradman A, Eskenazi B, Sutton P, Athanasoulis M, GoLdman LR. Iron deficiency associated with higher blood lead in children living in contaminated environments. Environ Health Perspect 2001; 109: 107984.

15. Koller K, Brown T, Spurgeon A, Levy L. Recent developments in low-level lead exposure and inte- llectual impairment in children. Environ Health Perspect 2004; 112: 987-94.

16. Adams RD, Víctor M. Principles of neurology, $5^{\text {th }}$ Ed New York: McGraw-Hill; 1993; 1210-12.

17. Rubens O, Logina I, Kravale I, Eglite M, Donaghy M. Peripheral neuropathy in chronic occupational inorganic lead exposure: a clinical and electrophysiological study. J Neurol Neurosurg Psychiatry 2001; 71: 200-4.

18. Rusyniak D. Pearls and Pitfalls in the approach to patients with neurotoxic sindromes. Semin Neurol 2001; 21: 407-16.

19. Stokes L, Letz R, Gerr F, Kolczak M, Mcneill, Chettle D, Kaye W. Neurotoxicity in young adults 20 years after childhood exposure to lead: the Bunker Hill experience. Occup Environ Med 1998; 55: 507-16.

20. Rogan WJ, Dietrich KN, Ware JH, Dockery DW, SalganiK M, Radcliffe J ET al. The effect of chelation therapy with succimer on neuropsychological development in children exposed to lead. N Engl J Med 2001; 344: 1421-6.

21. Liu X, Dietrich K, Radcliffe J, Ragan B, Rhoads G, Rogan W. Do children with falling blood lead levels have improved cognition? Pediatrics 2002; 110: 78791.

22. LidSKY T, SchNeIDER J. Lead neurotoxicity in children: basic mechanisms and clinical correlates. Brain 2003; 126: 5-19.

23. Johnston MV, Goldstein GW. Selective vulnerability of the developing brain to lead. Curr opin Neurol 1998; 11: 689-93.

24. Brown LL, SCheider JS, Linsky TI. Sensory and cognitive functions of the basal ganglia. Curr Opin Neurobiol 1997; 7: 157-63.

25. Fuster JM. The prefrontal cortex - An update: Time is of essence. Neuron 2001; 30: 319-33.

26. Trope I, López Villegas D, Cecil KM, Lenkinski RE. Exposure to lead appears to selectively alter metabolism of cortical gray matter. Pediatrics 2001; 107: 1437-42.

27. Vega J, Salinas J, Gutiérrez M, Mandamiento R, Yara M, Ponce J, Castro J. Lead levels and cognitive abilities in peruvian children. Rev Bras Psiquiatr 2006; 28 : 33-9.

28. Schwartz J. Lead, blood pressure, and cardiovascular disease in men. Arch Environ Health 1995; 50: 31-7.

29. Nash D, Magder L, Lustberg M, Sherwin R, Rubin R, Kaufmann R ET al. Blood lead, blood pressure, and hypertension in perimenopausal and postmenopausal women. JAMA 2003; 289: 1523-32.

30. Hu H. A 50-year follow-up of childhood plumbism. Hypertension, renal function, and hemoglobin levels among survivors. Am J Dis Child 1991; 145 : 681-7. 
31. Moss Me, Lanphear BP, Auinger P. Association of dental caries and blood lead levels among the US population. JAMA 1999; 281: 2294-8.

32. Selevan S, Rice D, Hogan K, Euling S, PfahlesHutchens, Bethel J. Blood lead concentration and delayed puberty in girls. N Engl J Med 2003; 348: 1527-36.

33. Benoff S, Jacob A, Hurley I. Male infertility and enviromental exposure to lead and cadmium. Hum Reprod Update 2000; 6: 107-21.

34. Borja-Aburto VH, Hertz-Picciotto i, Rojas López M, Farías P, Ríos C, Blanco J. Blood lead levels measu- red prospectively and risk of spontaneous abortion. Am J Epidemiol 1999; 150: 590-7.

35. Lin JL, Lin-Tan DT, Kuang-Hung H, Chun-Chen Y. Enviromental lead exposure and progression of chronic renal diseases in patients without diabetes. $\mathrm{N}$ Engl J Med 2003; 348: 277-86.

36. Schaumberg Da, Mendes F, Balaram M, Dana Mr, Sparrow $\mathrm{D}, \mathrm{Hu} \mathrm{H}$. Accumulated lead exposure and risk of agerelated cataract in men. JAMA 2004; 292: 2750-4.

37. Solimano G, Mazzei M. ¿De qué mueren los chilenos hoy?: perspectivas para el largo plazo. Rev Méd Chile 2007; 135: 932-8. 